\title{
On the Timing Option in a Futures Contract
}

\author{
Francesca Biagini, \\ Mathematics Institute \\ University of Munich \\ Theresienstr. 39 \\ D-80333 Munich, Germany \\ phone: +39-051-2094459
}

Francesca.Biagini@mathematik.uni-muenchen.de

\author{
Tomas Björk * \\ Department of Finance \\ Stockholm School of Economics \\ Box 6501, SE-113 83 Stockholm \\ SWEDEN \\ phone: +46-8-7369162 \\ tomas.bjork@hhs.se
}

(This version November 2005, first version June 2003)

To appear in

Mathematical Finance

\begin{abstract}
The timing option embedded in a futures contract allows the short position to decide when to deliver the underlying asset during the last month of the contract period. In this paper we derive, within a very general incomplete market framework, an explicit model independent formula for the futures price process in the presence of a timing option. We also provide a characterization of the optimal delivery strategy, and we analyze some concrete examples.
\end{abstract}

Key words: Futures contract, timing option, optimal stopping

${ }^{*}$ Corresponding author. Support from the Tom Hedelius and Jan Wallander Foundation is gratefully acknowledged. Both authors are grateful to B. Näslund, J. Kallsen, C. Kuehn, an anonymous associate editor, and an anonymous referee for a number of very helpful comments and suggestions. 


\section{Introduction}

In standard textbook treatments, a futures contract is typically defined by the properties of zero spot price and continuous (or discrete) resettlement, plus a simple no arbitrage condition at the last delivery day. If the underlying price process is denoted by $X_{t}$ and the futures price process for delivery at $T$ is denoted by $F(t, T)$ this leads to the well known formula

$$
F(t, T)=E^{Q}\left[X_{T} \mid \mathcal{F}_{t}\right], \quad 0 \leq t \leq T
$$

where $Q$ denotes the (not necessarily unique) risk neutral martingale measure.

In practice, however, there are a number of complicating factors which are ignored in the textbook treatment, and in particular it is typically the case that a standard futures contract has several embedded option elements. The most common of these options are the timing option, and the end-of-the-month option, the quality option, and the wild card option. All these options are options for the short end of the contract, and they work roughly as follows.

- The timing option is the option to deliver at any time during the last month of the contract.

- The end of the month option is the option to deliver at any day during the last week of the contract, despite the fact that the futures price for the last week is fixed on the first day of that week and then held constant.

- The quality option is the option to choose, out of a prespecified basket of assets, which asset to deliver.

- The wild card option is, for example for bond futures, the option to initiate delivery between 2 p.m. and 8 p.m. in the afternoon during the delivery month of the contract. The point here is that the futures price is settled at 2 p.m. but the trade in the underlying bonds goes on until 8 p.m.

The purpose of the present paper is to study the timing option within a very general framework, allowing for incomplete markets, and our goal is to investigate how the general formula (1) has to be modified when we introduce a timing option element. Our main result is given in Theorems 4.2 and 4.3 where it is shown that, independently of any model assumptions, the futures price in the presence of the timing option is given by the formula

$$
F(t, T)=\inf _{t \leq \tau \leq T} E^{Q}\left[X_{\tau} \mid \mathcal{F}_{t}\right] .
$$

where $\tau$ varies over the class of optional stopping times, and inf denotes the essential infimum. This formula is of course very similar to the pricing formula for an American option. Note, however, that (2) does not follow directly from standard theory for American contracts, the reason being that the futures price is not a price in the technical sense. The futures price process instead plays the role of the cumulative dividend process for the futures contract, which in turn can be viewed as a price-dividend pair, with spot price identically equal to zero. 
Furthermore we prove that the optimal delivery policy $\hat{\tau}(t)$, for a short contract entered at $t$, is given by

$$
\hat{\tau}=\inf \left\{t \geq 0 ; F(t, T)=X_{t}\right\} .
$$

We also study some special cases and show the following.

- If the underlying $X$ is the price of a traded financial asset without dividends, then it is optimal to deliver immediately, so $\hat{\tau}(t)=t$ and thus

$$
F(t, T)=X_{t} .
$$

- If the underlying $X$ has a convenience yield which is greater than the short rate, then the optimal delivery strategy is to wait until the last day. In this case we thus have $\hat{\tau}(t)=T$ and

$$
F(t, T)=E^{Q}\left[X_{T} \mid \mathcal{F}_{t}\right],
$$

which we recognize from (1) as the classical formula for futures contracts without a timing option.

Option elements of futures contract have also been studied earlier. The quality option is discussed in detail in Gay and Manaster (1984), and the wild card option is analyzed in Cohen (1995) and Gay and Manaster (1986). The timing option is (among other topics) treated in Boyle (1989) but theoretical results are only obtained for the special case when $X$ is the price process of a traded underlying asset. In this setting, and under the added assumption of a constant short rate, the formula (4) is derived.

The organization of the paper is as follows. In Section 2 we set the scene for the financial market. Note that we make no specific model assumptions at all about market completeness or the nature of the underlying process, and our setup allows for discrete as well as continuous time models. In Section 3 we derive a fundamental equation, the solution of which will determine the futures price process. We attack the fundamental equation by first studying the discrete time case in Section 4.1, and prove the main formula (2). In Section 4.2 we prove the parallel result in the technically more demanding continuous time case. We finish the main paper by some concrete financial applications, and in particular we clarify completely under which conditions the futures price process, including an embedded timing option, coincides with the classical formula (1). At the other end of the spectrum, we also investigate under which conditions immediate delivery is optimal.

\section{Setup}

We consider a financial market living on a stochastic basis $(\Omega, \mathcal{F}, \mathbf{F}, Q)$, where the filtration $\mathbf{F}=\left\{\mathcal{F}_{t}\right\}_{0<t<T}$ satisfies the usual conditions. We allow for both discrete and continuous time, so the contract period is either the interval $[0, T]$ or 
the set $\{0,1, \ldots, T\}$. To set the financial scene we need some basic assumptions, so for the rest of the paper we assume that there exists a predictable short rate process $r$, and a corresponding money account process $B$. In continuous time $B$ has the dynamics

$$
d B_{t}=r_{t} B_{t} d t
$$

In the discrete time case, the short rate at time $t$ will be denoted by $r_{t+1}$ so the bank account $B$ has the dynamics

$$
B_{t+1}=\left(1+r_{t+1}\right) B_{t}
$$

In this case the short rate is assumed to be predictable, i.e. $r_{t}$ is $\mathcal{F}_{t-1}$-measurable $\left(r_{t}\right.$ is known already at $t-1$ ) for all $t$, with the convention $\mathcal{F}_{-1}=\mathcal{F}_{0}$.

The market is assumed to be free of arbitrage in the sense the measure $Q$ above is a martingale measure w.r.t the money account $B$ for the given time horizon. Note that we do not assume market completeness. Obviously; if the market is incomplete, the martingale measure $Q$ will not be unique, so in an incomplete setting the pricing formulas derived below will depend upon the particular martingale measure chosen. We discuss this in more detail in Section 5.

We will need a weak boundedness assumption on the short rate.

Assumption 2.1 For the rest of the paper we assume the following.

- In the continuous time case we assume that the interest rate process is predictable, and that there exists a positive real number c such that

$$
r_{t} \geq-c
$$

with probability one, for all t.

- Defining the money account as usual by $B$ by $B_{t}=\exp \left(\int_{0}^{t} r_{s} d s\right)$ we assume that

$$
E^{Q}\left[B_{T}\right]<\infty
$$

- In the discrete time case we assume the interest rate process is predictable, and that there exists a positive real number $c$ such that

$$
1+r_{n} \geq c
$$

with probability one, for all $n$.

Remark 2.1 We note that the if we define $C$ by

$$
C_{t}=\sup _{t \leq \tau \leq T} E^{Q}\left[B_{\tau} \mid \mathcal{F}_{t}\right]
$$

where $\tau$ varies over the class of stopping times, then the inequality $E^{Q}\left[B_{T}\right]<\infty$ easily implies

$$
C_{t}<\infty
$$

$Q-$ a.s. for all $t \in[0, T]$. 
Within this framework we now want to consider a futures contract with an embedded timing option.

Assumption 2.2 We assume the existence of an exogenously specified nonnegative adapted cadlag process $X$. The process $X$ will henceforth be referred to as the index process, and we assume that

$$
E^{Q}\left[X_{t}\right]<\infty, \quad 0 \leq t \leq T
$$

The interpretation of this assumption is that the index process $X$ is the underlying process on which the futures contract is written.

For obvious reasons we want to include contracts like commodity futures, index futures, futures with an embedded quality option, and also futures on a non-financial index like a weather futures contract. For this reason we do not assume that $X$ is the price process of a traded financial asset in an idealized frictionless market. Typical choices of $X$ could thus be one of the following.

- $X_{t}$ is the price at time $t$ of a commodity, with a non trivial convenience yield.

- $X_{t}$ is the price at time $t$ of a, possibly dividend paying, financial asset.

- $X_{t}=\min \left\{S_{t}^{1}, \ldots, S_{t}^{n}\right\}$ where $S_{t}^{1}, \ldots, S_{t}^{n}$ are price processes of financial assets (for example stocks or bonds). This setup would be natural if we have an embedded quality option.

- $X_{t}$ is a non financial process, like the temperature at some prespecified location.

We now want to define a futures contract, with an embedded timing option, on the underlying index process $X$ over the time interval $[0, T]$. If, for example, we are considering a US interest rate future, this means that the interval $[0, T]$ corresponds to the last month of the contract period. Note that we thus assume that the timing option is valid for the entire interval $[0, T]$. The analysis of the futures price process for times prior to the timing option period, is trivial and given by standard theory. If, for example, we let the timing option be active only in the interval $\left[T_{0}, T\right]$, then we immediately obtain

$$
F(t, T)=E^{Q}\left[F\left(T_{0}, T\right) \mid \mathcal{F}_{t}\right], \quad 0 \leq t \leq T_{0},
$$

where $F\left(T_{0}, T\right)$ is given by the theory developed in the present paper. We can now give formal definition of the (continuous time) contract. See below for the discrete time modification.

Definition 2.1 A futures contract on $X$ with final delivery date $T$, including an embedded timing option, on the interval $[0, T]$, with continuous resettlement, is a financial contract satisfying the following clauses. 
- At each time $t \in[0, T]$ there exists on the market a futures price quotation denoted by $F(t, T)$. Furthermore, for each fixed $T$, the process $t \longmapsto F(t, T)$ is a semimartingale w.r.t. the filtration $\mathbf{F}$. Since $T$ will be fixed in the discussion below, we will often denote $F(t, T)$ by $F_{t}$.

- The holder of the short end of the futures contract can, at any time $t \in$ $[0, T]$, decide whether to deliver or not. The decision whether to deliver at $t$ or not is allowed to be based upon the information contained in $\mathcal{F}_{t}$.

- If the holder of short end decides to deliver at time $t$, she will pay the amount $X_{t}$ and receive the quoted futures price $F(t, T)$.

- If delivery has not been made prior to the final delivery date $T$, the holder of the short end will pay $X_{T}$ and receive $F(T, T)$.

- During the entire interval $[0, T]$ there is continuous resettlement as for a standard futures contract. More precisely; over the infinitesimal interval $[t, t+d t]$ the holder of the short end will pay the amount

$$
d F(t, T)=F(t+d t, T)-F(t, T) .
$$

- The spot price of the futures contract is always equal to zero, i.e. you can at any time enter or leave the contract at zero cost.

- The cash flow for the holder of the long end is the negative of the cash flow for the short end.

The important point to notice here is that the timing option is only an option for the holder of the short end of the contract. For discrete time models, the only difference is the resettlement clause which then says that if you hold a short future between $t$ and $t+1$, you will pay the amount $F(t+1, T)-F(t, T)$ at time $t+1$.

Our main problem is the following.

Problem 2.1 Given an exogenous specification of the index process $X$, what can be said about the existence and structure of the futures price process $F(t, T)$ ?

\section{The Fundamental Pricing Equation}

We now go on to reformulate Problem 2.1 in more precise mathematical terms, and this will lead us to a fairly complicated infinite dimensional system of equations for the determination of the futures price process (if that object exists). We focus on the continuous time case, the discrete time case being very similar. 


\subsection{The Pricing Equation in Continuous Time}

For the given final delivery date $T$, let us consider a fixed point in time $t \leq T$ and discuss the (continuous time) futures contract from the point of view of the short end of the contract. From the definition above, it is obvious that the holder of the short end has to decide on a delivery strategy, and we formalize such a strategy as a stopping time $\tau$, with $t \leq \tau \leq T, Q-$ a.s. If the holder of the short end uses the particular delivery strategy $\tau$, then the arbitrage free value of her cash flows is given by the expression

$$
E^{Q}\left[e^{-\int_{t}^{\tau} r_{s} d s}\left(F_{\tau}-X_{\tau}\right)-\int_{t}^{\tau} e^{-\int_{t}^{u} r_{s} d s} d F_{u} \mid \mathcal{F}_{t}\right] .
$$

The first term in the expectation corresponds to the cash flow for the actual delivery, i.e. the short end delivers $X_{\tau}$ and receives the quoted futures price $F_{\tau}$, and the integral term corresponds to the cash flow of the continuous resettlement. Since the timing option is an option for the holder of the short end, she will try to choose the stopping time $\tau$ so as to maximize the arbitrage free value. Thus, the value of the short end of the futures contract at time $t$ is given by

$$
\sup _{t \leq \tau \leq T} E^{Q}\left[e^{-\int_{t}^{\tau} r_{s} d s}\left(F_{\tau}-X_{\tau}\right)-\int_{t}^{\tau} e^{-\int_{t}^{u} r_{s} d s} d F_{u} \mid \mathcal{F}_{t}\right],
$$

where, for short sup denotes the essential supremum. We now recall that, by definition, the spot price of the futures contract is always equal to zero, and we have thus derived our fundamental pricing equation, which is in fact an equilibrium condition for each $t$.

Proposition 3.1 The futures price process $F$, if it exists, will satisfy for each $t \in[0, T]$, the fundamental pricing equation

$$
\sup _{t \leq \tau \leq T} E^{Q}\left[e^{-\int_{t}^{\tau} r_{s} d s}\left(F_{\tau}-X_{\tau}\right)-\int_{t}^{\tau} e^{-\int_{t}^{u} r_{s} d s} d F_{u} \mid \mathcal{F}_{t}\right]=0,
$$

where $\tau$ varies over the class of stopping times.

Some remarks are now in order.

\section{Remark 3.1}

- At first sight, equation (17) may look like a standard optimal stopping problem, but it is in fact more complicated than that. Obviously: for a given futures price process $F$, the left hand side of (17) represents a standard optimal stopping problem, but the point here is that the futures process $F$ is not an a priori given object. Instead we have to find a process $F$ such that the optimal stopping problem defined by the left hand side of (3) has the optimal value zero for each $t \leq T$. Thus, our formalized problem is as follows. 
- It is not at all obvious that there exists a solution process $F$ to the fundamental equation 3.1, and it is even less obvious that a solution will be unique. These questions will be treated below.

- It may seem that we are only considering the futures price process from the perspective of the seller of the contract. However; the total cash flows sum to zero, so if the fundamental pricing equation above is satisfied, the (spot) value of the contract is zero also to the buyer (and if exercised in a non optimal fashion, the value would be positive for the buyer and negative for the seller).

The main problems to be studied are the following.

Problem 3.1 Consider an exogenously given index process $X$.

- Our primary problem is to find a process $\left\{F_{t} ; \quad 0 \leq t \leq T\right\}$ such that (17) is satisfied for all $t \in[0, T]$.

- If we manage to find a process $F$ with the above properties, we would also like to find, for each fixed $t \in[0, T]$, the optimal stopping time $\hat{\tau}_{t}$ realizing the supremum in

$$
\sup _{t \leq \tau \leq T} E^{Q}\left[e^{-\int_{t}^{\tau} r_{s} d s}\left(F_{\tau}-X_{\tau}\right)-\int_{t}^{\tau} e^{-\int_{t}^{u} r_{s} d s} d F_{u} \mid \mathcal{F}_{t}\right] .
$$

We also note that even if we manage to prove the existence of a solution process $F$, there is no guarantee of the existence of an optimal stopping time $\hat{\tau}_{t}$, since in the general case we can (as usual) only be sure of the existence of $\epsilon$-optimal stopping times.

\subsection{Some Preliminary Observations}

A complete treatment of the pricing equation will be given in the next two sections, but we may already at this stage draw some preliminary conclusions.

Lemma 3.1 The futures price process has to satisfy the condition

$$
\begin{aligned}
F(t, T) & \leq X_{t}, \quad \forall t \leq T, \\
F(T, T) & =X_{T} .
\end{aligned}
$$

Proof. The economic reason for (19) is obvious. If, for some $t$, we have $F(t, T)>X_{t}$ then we enter into a short position (at zero cost) and immediately decide to deliver. We pay $X_{t}$ and receive $F(t, T)$ thus making an arbitrage profit, and immediately close the position (again at zero cost).

A more formal proof is obtained by noting that the fundamental equation (17) implies that

$$
E^{Q}\left[e^{-\int_{t}^{\tau} r_{s} d s}\left(F_{\tau}-X_{\tau}\right)-\int_{t}^{\tau} e^{-\int_{t}^{u} r_{s} d s} d F_{u} \mid \mathcal{F}_{t}\right] \leq 0,
$$


for all stopping times $\tau$ with $t \leq \tau \leq T$. In particular, the (21) holds for $\tau=t$ which gives us

$$
E^{Q}\left[F_{t}-X_{t} \mid \mathcal{F}_{t}\right] \leq 0
$$

and since both $F$ and $X$ are adapted, the inequality (19) follows. The boundary condition (20) is an immediate consequence of no arbitrage.

We finish this section by proving that for the very special case of zero short rate, we can easily obtain an explicit formula for the futures price process. Note that, for simplicity of notation, the symbol inf henceforth denotes the essential infimum.

Proposition 3.2 If $r \equiv 0$, then

$$
F(t, T)=\inf _{t \leq \tau \leq T} E^{Q}\left[X_{\tau} \mid \mathcal{F}_{t}\right]
$$

Proof. With zero short rate the fundamental equation reads as

$$
\sup _{t \leq \tau \leq T} E^{Q}\left[\left(F_{\tau}-X_{\tau}\right)-\int_{t}^{\tau} d F_{u} \mid \mathcal{F}_{t}\right]=0
$$

Using the fact that $\int_{t}^{\tau} d F_{u}=F_{\tau}-F_{t}$ we thus obtain

$$
\sup _{t \leq \tau \leq T} E^{Q}\left[F_{t}-X_{\tau} \mid \mathcal{F}_{t}\right]=0 .
$$

Since $F$ is adapted this implies

$$
F_{t}=-\sup _{t \leq \tau \leq T} E^{Q}\left[-X_{\tau} \mid \mathcal{F}_{t}\right]=\inf _{t \leq \tau \leq T} E^{Q}\left[X_{\tau} \mid \mathcal{F}_{t}\right]
$$

In the Section 4 we will prove that the formula (23) is in fact valid also in the general case without the assumption of zero short rate.

\subsection{The Pricing Equation in Discrete Time Case}

By going through a completely parallel argument as above, it is easy to see that in a discrete time model the fundamental equation (17) will have the form

$$
\sup _{t \leq \tau \leq T} E^{Q}\left[\left(\prod_{n=t+1}^{\tau} \frac{1}{1+r_{n}}\right)\left(F_{\tau}-X_{\tau}\right)-\sum_{n=t+1}^{\tau}\left(\prod_{u=t+1}^{n} \frac{1}{1+r_{u}}\right) \Delta F_{n} \mid \mathcal{F}_{t}\right]=0
$$

where $\Delta F_{n}=F_{n}-F_{n-1}$.

\section{Determining the Futures Price Process}

In this section we will solve the fundamental pricing equations (17) and (27), thus obtaining an explicit representation for the futures price process. We start with the discrete time case, since this is technically less complicated. 


\subsection{The Discrete Time Case}

We will not analyze equation (27) directly, but rather use a standard dynamic programming argument as a way of attacking the problem.

To do this we consider the decision problem of the holder of the short end of the futures contract. Suppose that at time $n$ you have entered into the short contract. Then you have the following two alternatives:

1. You can decide to deliver immediately, in which case you will receive the amount

$$
F_{n}-X_{n}
$$

2. You can decide to wait until $n+1$. This implies that at time $n+1$ you will obtain the amount $F_{n}-F_{n+1}$. The arbitrage free value, at $n$, of this cash flow is given by the expression

$$
E^{Q}\left[\frac{F_{n}-F_{n+1}}{1+r_{n+1}} \mid \mathcal{F}_{n}\right]=\frac{1}{1+r_{n+1}} E^{Q}\left[F_{n}-F_{n+1} \mid \mathcal{F}_{n}\right],
$$

where we have used the fact that $r$ is predictable. The value of your contract, after having received the cash flow above, is by definition zero.

Obviously you would like to make the best possible decision, so the value at time $n$ of a short position is given by

$$
\max \left[\left(F_{n}-X_{n}\right), \frac{1}{1+r_{n+1}} E^{Q}\left[F_{n}-F_{n+1} \mid \mathcal{F}_{n}\right]\right] .
$$

On the other hand, the spot price of the futures contract is by definition always equal to zero, so we conclude that

$$
\max \left[\left(F_{n}-X_{n}\right), \frac{1}{1+r_{n+1}} E^{Q}\left[F_{n}-F_{n+1} \mid \mathcal{F}_{n}\right]\right]=0,
$$

for $n=1, \ldots T-1$.

We now recall the following basic result from optimal stopping theory (see Snell 1952).

Theorem 4.1 (Snell Envelope Theorem) With notations as above, define the optimal value process $V$ by

$$
V_{t}=\inf _{t \leq \tau \leq T} E^{Q}\left[X_{\tau} \mid \mathcal{F}_{t}\right]
$$

where $\tau$ varies of the class of stopping times. Then $V$ is characterized by the property of being the largest submartingale dominated by $X$.

The process $V$ above is referred to as the (lower) Snell Envelope of $X$ with horizon $T$, and we may now state and prove our main result in discrete time. 
Theorem 4.2 Given the index process $X$, and a final delivery date $T$, the futures price process $F(t, T)$ exists uniquely and coincides with the lower Snell envelope of $X$ with horizon $T$, i.e.

$$
F(t, T)=\inf _{t \leq \tau \leq T} E^{Q}\left[X_{\tau} \mid \mathcal{F}_{t}\right],
$$

where $\tau$ varies over the set of stopping times. Furthermore, if the short position is entered at time $t$, then the optimal delivery time is given by

$$
\hat{\tau}(t)=\inf \left\{k \geq t ; F_{k}=X_{k}\right\} .
$$

Proof. We will show that there exists a unique futures price process $F$ and that it is in fact the largest submartingale dominated by $X$. The result then follows directly from the Snell Envelope Theorem.

We start by noting that, since by Assumption $2.11+r_{n}>0$, we can write (31) as

$$
\max \left[\left(F_{n}-X_{n}\right), E^{Q}\left[F_{n}-F_{n+1} \mid \mathcal{F}_{n}\right]\right]=0,
$$

and since $F$ is adapted this implies

$$
F_{n}=-\max \left[-X_{n},-E^{Q}\left[F_{n+1} \mid \mathcal{F}_{n}\right]\right] .
$$

This gives us the recursive system

$$
\begin{aligned}
& F_{n}=\min \left[X_{n}, E^{Q}\left[F_{n+1} \mid \mathcal{F}_{n}\right]\right], \quad n=0, \ldots, T-1 \\
& F_{T}=X_{T},
\end{aligned}
$$

where the boundary conditions follows directly from no arbitrage. This recursive formula for $F$ proves existence and uniqueness.

We now go on to prove that $F$ is a submartingale dominated by $X$. From (37) we immediately have

$$
F_{n} \leq E^{Q}\left[F_{n+1} \mid \mathcal{F}_{n}\right],
$$

which proves the submartingale property, and we also have

$$
F_{n} \leq X_{n},
$$

which in fact was already proved in Lemma 3.1.

It remains to prove the maximality property of $F$ and for this we use backwards induction. Assume thus that $Z$ is a submartingale dominated by $X$. In particular this implies that $Z_{T} \leq X_{T}$, but since $F_{T}=X_{T}$ we obtain $Z_{T} \leq F_{T}$. For the induction step, assume that $Z_{n+1} \leq F_{n+1}$. We then want to prove that this implies the inequality $Z_{n} \leq F_{n}$. To do this we observe that the submartingale property of $Z$ together with the induction assumption implies

$$
Z_{n} \leq E^{Q}\left[Z_{n+1} \mid \mathcal{F}_{n}\right] \leq E^{Q}\left[F_{n+1} \mid \mathcal{F}_{n}\right]
$$

By assumption we also have $Z_{n} \leq X_{n}$, so we have in fact

$$
Z_{n} \leq \min \left[X_{n}, E^{Q}\left[F_{n+1} \mid \mathcal{F}_{n}\right]\right],
$$

and from this inequality and (37) we obtain $Z_{n} \leq F_{n}$. 


\subsection{The Continuous Time Case and Some Examples}

We now go on to find a formula for the futures price process in continuous time and, based on the discrete time results of the previous section, we of course conjecture that also in continuous time we have the formula $F(t, T)=$ $\inf _{t \leq \tau \leq T} E^{Q}\left[X_{\tau} \mid \mathcal{F}_{t}\right]$. Happily enough, this also turns out to be correct, but a technical problem is that in continuous time it is impossible to just mimic the discrete time arguments above, since we can no longer use induction. Thus we have to use other methods, and we will rely on some very nontrivial results from continuous time optimal stopping theory. All these results can be found in the highly readable Appendix D in Karatzas and Shreve (1998).

From now on, we assume the following further integrability condition on the underlying process $X$ :

$$
E^{Q}\left[\sup _{0 \leq t \leq T} X_{t}\right]<\infty
$$

Before proving our main result, we need the following technical result.

Lemma 4.1 Suppose that the index process $X_{t}$ satisfies condition (39) and consider its Snell Envelope $F(t, T)=\inf _{t \leq \tau \leq T} E^{Q}\left[X_{\tau} \mid \mathcal{F}_{t}\right]$. Let $H_{t}=e^{-\int_{0}^{t} r_{u} d u}$, where $r_{t}$ satisfies the weak boundness Assumption 2.1. If $\tau \leq T$ is a stopping time such that the stopped submartingale $F^{\tau}$ is a martingale, then the stochastic integral $\int_{0}^{t} e^{-\int_{0}^{s} r_{u} d u} d F_{s}^{\tau}$ is a martingale.

Proof. We recall that by Assumption 2.2, the index process $X_{t}$ is supposed to be a nonnegative adapted càdlàg process. Hence if $\tau$ is a stopping time such that the stopped submartingale $F^{\tau}$ is a martingale, it is indeed a càdlàg martingale and consequently we need only to verify that

$$
E^{Q}\left[\left(\int_{0}^{T} H_{s}^{2} d\left[F^{\tau}\right]_{s}\right)^{\frac{1}{2}}\right]<\infty
$$

in order to guarantee that the stochastic integral $\int_{0}^{t} e^{-\int_{0}^{s} r_{u} d u} d F_{s}^{\tau}$ is a martingale by using the Burkholder-Davis-Gundy inequalities (see Revuz and Yor (1994), p.151, and Protter (2004) p.193). Here, the process $\left[F^{\tau}\right]_{t}$ is the quadratic variation of $F^{\tau}$ (for further details, see Protter (2004) p.66). Since $H_{t}=$ $e^{-\int_{0}^{t} r_{u} d u}$ and $r_{t}$ is uniformly bounded from below (Assumption 2.1), we obtain the following estimates

$$
\begin{aligned}
E^{Q}\left[\left(\int_{0}^{T} H_{s}^{2} d\left[F^{\tau}\right]_{s}\right)^{\frac{1}{2}}\right] & =E^{Q}\left[\left(\int_{0}^{T} e^{-2 \int_{0}^{s} r_{u} d u} d\left[F^{\tau}\right]_{s}\right)^{\frac{1}{2}}\right] \\
& \leq E^{Q}\left[\left(\int_{0}^{T} e^{2 c s} d\left[F^{\tau}\right]_{s}\right)^{\frac{1}{2}}\right]
\end{aligned}
$$




$$
\leq e^{c T} E^{Q}\left[\left(\left[F^{\tau}\right]_{T}\right)^{\frac{1}{2}}\right]
$$

Since the process $F$ is given by the Snell Envelope of $X$, it is a nonnegative submartingale dominated by $X$, hence we can use the Burkholder-Davis-Gundy inequalities and get

$$
E^{Q}\left[\left(\left[F^{\tau}\right]_{T}\right)^{\frac{1}{2}}\right] \leq k E^{Q}\left[\sup _{0 \leq t \leq T} F_{t}^{\tau}\right] \leq k E^{Q}\left[\sup _{0 \leq t \leq T} F_{t}\right] \leq k E^{Q}\left[\sup _{0 \leq t \leq T} X_{t}\right]
$$

where $k$ is a suitable constant. Since $X$ satisfies (39), the last term of the inequality is finite. Hence, we can conclude that the stochastic integral $\int_{0}^{t} e^{-\int_{0}^{s} r_{u} d u} d F_{s}^{\tau}$ is a martingale.

We may now state our main result in continuous time.

Theorem 4.3 Under Assumption 2.1 and if (39) holds, there exists, for each fixed $T$, a unique futures price process $F(t, T)$ solving the the fundamental equation (17). The futures price process is given by the expression

$$
F(t, T)=\inf _{t \leq \tau \leq T} E^{Q}\left[X_{\tau} \mid \mathcal{F}_{t}\right] .
$$

Furthermore, if $X$ has continuous trajectories, then the optimal delivery time $\hat{\tau}(t)$, for the holder of a short position at time $t$ is given by

$$
\hat{\tau}(t)=\inf \left\{u \geq t ; F(u, T)=X_{u}\right\} .
$$

Proof. We first show that if we define $F$ by (41) then $F$ solves the pricing equation (17). Having proved this we will then go on to prove that if $F$ solves (17), then $F$ must necessarily have the form (41).

We thus start by defining a process $F_{t}$ as the lower Snell envelope of $X$, i.e.

$$
F_{t}=\inf _{t \leq \tau \leq T} E^{Q}\left[X_{\tau} \mid \mathcal{F}_{t}\right]
$$

and we want to show that for this choice of $F$, the fundamental pricing equation (17) is satisfied. From the (continuous time version of) Snell Envelope Theorem, we know that $F$ is a submartingale. Thus (for fixed $t$ ) the stochastic differential

$$
e^{-\int_{t}^{u} r_{s} d s} d F_{u}
$$

is a submartingale differential, and since $F \leq X$ we see that the inequality

$$
E^{Q}\left[e^{-\int_{t}^{\tau} r_{s} d s}\left(F_{\tau}-X_{\tau}\right)-\int_{t}^{\tau} e^{-\int_{t}^{u} r_{s} d s} d F_{u} \mid \mathcal{F}_{t}\right] \leq 0,
$$

will hold for every stopping time $\tau$ with $t \leq \tau \leq T$. To show that $F$ defined as above satisfies (17) it is therefore enough to show that for some stopping time $\tau$ we have

$$
E^{Q}\left[e^{-\int_{t}^{\tau} r_{s} d s}\left(F_{\tau}-X_{\tau}\right)-\int_{t}^{\tau} e^{-\int_{t}^{u} r_{s} d s} d F_{u} \mid \mathcal{F}_{t}\right]=0 .
$$


For simplicity of exposition we now assume that, for each $t$, the infimum in the optimal stopping problem

$$
\inf _{t \leq \tau \leq T} E^{Q}\left[X_{\tau} \mid \mathcal{F}_{t}\right]
$$

is realized by some (not necessarily unique) stopping time $\hat{\tau}_{t}$. The proof of the general case is more complicated and therefore relegated to the appendix. From general theory (see Karatzas and Shreve (1998) p. 355, Theorem D9) we cite the following facts.

1. With $F$ defined by (43) we have

$$
F_{\hat{\tau}_{t}}=X_{\hat{\tau}_{t}} .
$$

2. The stopped process $F^{\hat{\tau}_{t}}$ defined by

$$
F_{s}^{\hat{\tau}_{t}}=F_{s \wedge \hat{\tau}_{t}}
$$

where $\wedge$ denotes the minimum, is a martingale on the interval $[t, T]$.

Choosing $\tau=\hat{\tau}_{t}$, equation (45) thus reduces to the equation

$$
E^{Q}\left[\int_{t}^{\hat{\tau}_{t}} e^{-\int_{t}^{u} r_{s} d s} d F_{u} \mid \mathcal{F}_{t}\right]=0
$$

which we can write as

$$
E^{Q}\left[\int_{t}^{T} e^{-\int_{t}^{u} r_{s} d s} d F_{u}^{\hat{\tau}_{t}} \mid \mathcal{F}_{t}\right]=0
$$

and since, by a small variation of Lemma 4.1 , the process $\int_{t}^{s} e^{-\int_{t}^{u} r_{v} d v} d F_{u}^{\hat{\tau}_{t}}$ is a martingale, (50) is indeed satisfied. This proves existence.

In order to prove uniqueness let us assume that, for a fixed $T$, a process $F$ solves (17). We now want to prove that $F$ is in fact the lower Snell envelope of $X$, i.e. we have to prove that $F$ is the largest submartingale dominated by $X$.

We first note that, after premultiplication with the exponential factor

$$
e^{-\int_{0}^{t} r_{s} d s}
$$

the fundamental equation (17) can be rewritten as

$$
\int_{0}^{t} e^{-\int_{0}^{u} r_{s} d s} d F_{u}=\inf _{t \leq \tau \leq T} E^{Q}\left[\int_{0}^{\tau} e^{-\int_{0}^{u} r_{s} d s} d F_{u}+e^{-\int_{0}^{\tau} r_{s} d s}\left(X_{\tau}-F_{\tau}\right) \mid \mathcal{F}_{t}\right]
$$

Defining the process $V$ by

$$
V_{t}=\int_{0}^{t} e^{-\int_{0}^{u} r_{s} d s} d F_{u},
$$


we thus see that $V$ is the lower Snell envelope of the process $Z$, defined by

$$
Z_{t}=\int_{0}^{t} e^{-\int_{0}^{u} r_{s} d s} d F_{u}+e^{-\int_{0}^{t} r_{s} d s}\left(X_{t}-F_{t}\right)
$$

i.e.

$$
V_{t}=\inf _{t \leq \tau \leq T} E^{Q}\left[Z_{\tau} \mid \mathcal{F}_{t}\right]
$$

From the Snell Theorem it now follows that $V$ is a submartingale, and since the exponential integrand in (52) is positive, this implies that also $F$ is a submartingale. We have already proved in Proposition 3.1 that $F \leq X$ so it only remains to prove maximality. To this end, let us assume that $G$ is a submartingale dominated by $X$. We now want to prove that $G_{t} \leq F_{t}$ for every $t \leq T$. To this end we choose a fixed but arbitrary $t$. For simplictiy of exposition we now assume that, for a fixed $t$, there exists and optimal stopping time attaining the infimum in (54), and the denote this stopping time by $\bar{\tau}_{t}$. The proof in the general case is found in the appendix. We obtain from (17)

$$
E^{Q}\left[e^{-\int_{t}^{\bar{\tau}_{t}} r_{s} d s}\left(F_{\bar{\tau}_{t}}-X_{\bar{\tau}_{t}}\right)-\int_{t}^{\bar{\tau}_{t}} e^{-\int_{t}^{u} r_{s} d s} d F_{u} \mid \mathcal{F}_{t}\right]=0
$$

Since $F \leq X$ and $F$ is a submartingale, this implies that

$$
F_{\bar{\tau}_{t}}=X_{\bar{\tau}_{t}}
$$

and that

$$
E^{Q}\left[\int_{t}^{\bar{\tau}_{t}} e^{-\int_{t}^{u} r_{s} d s} d F_{u} \mid \mathcal{F}_{t}\right]=0
$$

which in turn (after premultiplication by an exponential factor) implies that

$$
E^{Q}\left[V_{\bar{\tau}_{t}} \mid \mathcal{F}_{t}\right]=V_{t}
$$

Since $V$ is a submartingale, this implies that the stopped process $V^{\bar{\tau}_{t}}$ is in fact a martingale on the time interval $[t, T]$, which in turn implies that the stopped process $F^{\bar{\tau}_{t}}$ is a martingale on $[t, T]$. In particular we then have

$$
F_{t}=E^{Q}\left[F_{\bar{\tau}_{t}} \mid \mathcal{F}_{t}\right]=E^{Q}\left[X_{\bar{\tau}_{t}} \mid \mathcal{F}_{t}\right]
$$

where we have used (56). On the other hand, from the assumptions on $G$ we have

$$
G_{t} \leq E^{Q}\left[G_{\bar{\tau}_{t}} \mid \mathcal{F}_{t}\right] \leq E^{Q}\left[X_{\bar{\tau}_{t}} \mid \mathcal{F}_{t}\right]=F_{t}
$$

which proves the maximality of $F$.

The second statement in the theorem formulation follows directly from Theorem D.12 in Karatzas and Shreve (1998).

As a more or less trivial consequence, we immediately have the following result for futures on underlying sub- and supermartingales. 
Proposition 4.1 If $X$ is a submartingale under $Q$, then

$$
F(t, T)=X_{t},
$$

and it is always optimal to deliver at once, i.e.

$$
\hat{\tau}(t)=t .
$$

If $X$ is a supermartingale under $Q$, then

$$
F(t, T)=E^{Q}\left[X_{T} \mid \mathcal{F}_{t}\right],
$$

and it is always optimal to wait, i.e.

$$
\hat{\tau}(t)=T .
$$

Proof. Follows at once from the representation (41).

From this result we immediately have some simple financial implications.

Proposition 4.2 Assume that one of the following conditions hold

1. $X$ is the price process of a traded financial asset without dividends, and the short rate process $r$ is nonnegative with probability one.

2. $X$ is the price process of a traded asset with a continuous dividend yield rate process $\delta$ such that $\delta_{t} \leq r_{t}$ for all $t$ with probability one.

3. $X$ is an exchange rate process (quoted as units of domestic currency per unit of foreign currency) and the foreign short rate $r^{f}$ has the property that $r_{t}^{f} \leq r_{t}$ for all $t$ with probability one.

Then the futures price is given by

$$
F(t, T)=X_{t},
$$

and it is always optimal to deliver at once, i.e.

$$
\hat{\tau}(t)=t .
$$

Proof. The $Q$ dynamics of $X$ are as follows in the three cases above

$$
\begin{aligned}
d X_{t} & =r_{t} X_{t} d t+d M_{t}, \\
d X_{t} & =X_{t}\left[r_{t}-\delta_{t}\right] d t+d M_{t}, \\
d X_{t} & =X_{t}\left[r_{t}-r_{t}^{f}\right] d t+d M_{t},
\end{aligned}
$$

where $M$ is the generic notation for a martingale. The assumptions guarantee, in each case, that $X$ is a $Q$-submartingale and we may thus apply Proposition 4.1 .

With an almost identical proof we have the following parallel result, which shows that under certain conditions the futures price process is not changed by the introduction of a timing option. 
Proposition 4.3 Assume that one of the following conditions hold

1. $X$ is the price process of an asset with a convenience yield rate process $\gamma$ such that $\gamma_{t} \geq r_{t}$ for all $t$ with probability one.

2. $X$ is an exchange rate process (quoted as units of domestic currency per unit of foreign currency) and the foreign short rate $r^{f}$ has the property that $r_{t}^{f} \geq r_{t}$ for all $t$ with probability one.

Then the futures price is given by

$$
F(t, T)=E^{Q}\left[X_{T} \mid \mathcal{F}_{t}\right],
$$

and it is always optimal to wait until $T$ to deliver, i.e.

$$
\hat{\tau}(t)=T .
$$

\section{Conclusions and Discussion}

The main result of the present paper is given in Theorems 4.2 and 4.3 where we provide the formula

$$
F(t, T)=\inf _{t \leq \tau \leq T} E^{Q}\left[X_{\tau} \mid \mathcal{F}_{t}\right]
$$

which gives us the arbitrage free futures price process in terms of the underlying index $X$ and the martingale measure $Q$. In Section 4.2 we also gave some immediate implications of the general formula, but these results are of secondary importance. We now have a number of comments on the main result (69).

- We see that the formula (69) for the futures price in the presence of a timing option looks very much like the standard pricing formula for an American option. Therefore, one may perhaps expect that (69) is a direct consequence of the well known pricing formula for American contracts. As far as we can understand, this is not the case. As noted above, the "futures price process" $F(t, T)$ is not a price process at all, since its economic role is that of a cumulative dividend process for the futures contract (which always has spot price zero).

From a more technical point of view, we also see that the determination of the $F$ process is quite intricate, since $F$ has to solve the infinite dimensional fundamental equation (17) (which is in fact an equilibrium condition for each $t$ ) or the corresponding discrete time equation (31).

- We assumed absence of arbitrage but we did not make any assumptions concerning market completeness. In an incomplete market, the martingale measure $Q$ is not unique, so in this case formula (69) does not provide us with a unique arbitrage free futures price process. In an incomplete setting, the interpretation of Theorems 4.2 and 4.3 is then that, given absence of arbitrage, the futures price process has to be given by formula (69) for 
some choice of a martingale measure $Q$. This is of course completely parallel to the standard risk neutral pricing formula which, in the incomplete setting, gives us a price of a contingent claim which depends upon the martingale measure chosen. Note however, that some of the results above are independent of the choice of the martingale measure. In particular this is true for Proposition 4.2.

\section{A A Proof of Theorem 4.3 in the General Case}

In this Appendix we provide the proof of Theorem 4.3 for the general case, i.e. without assuming that the infima in (46) and (54) are attained.

We start with the existence proof and to this end we define the process $F$ (as before) by

$$
F_{t}=\inf _{t \leq \tau \leq T} E^{Q}\left[X_{\tau} \mid \mathcal{F}_{t}\right]
$$

and we have to show that $F$ thus defined satisfies the fundamental pricing equation

$$
\sup _{t \leq \tau \leq T} E^{Q}\left[e^{-\int_{t}^{\tau} r_{s} d s}\left(F_{\tau}-X_{\tau}\right)-\int_{t}^{\tau} e^{-\int_{t}^{u} r_{s} d s} d F_{u} \mid \mathcal{F}_{t}\right]=0 .
$$

As in the simplified proof above it is easy to see that

$$
E^{Q}\left[e^{-\int_{t}^{\tau} r_{s} d s}\left(F_{\tau}-X_{\tau}\right)-\int_{t}^{\tau} e^{-\int_{t}^{u} r_{s} d s} d F_{u} \mid \mathcal{F}_{t}\right] \leq 0
$$

for all stopping times $\tau$ with $t \leq \tau \leq T$. Thus; to prove that $F$ satisfies (71) it is enough to prove that there exists a sequence of stopping times $\left\{\tau_{n}\right\}_{n=1}^{\infty}$ such that

$$
E^{Q}\left[e^{-\int_{t}^{\tau_{n}} r_{s} d s}\left(F_{\tau_{n}}-X_{\tau_{n}}\right)-\int_{t}^{\tau_{n}} e^{-\int_{t}^{u} r_{s} d s} d F_{u} \mid \mathcal{F}_{t}\right] \geq-\frac{1}{n}
$$

for all $n$. To do this we consider a fixed $t$ and define $\tau_{n}$ by

$$
\tau_{n}=\inf \left\{s \geq t ; F_{s} \geq X_{s}(1-1 / n)\right\} .
$$

Like in the earlier proof we can rewrite the stochastic integral in (73) as

$$
\int_{t}^{\tau_{n}} e^{-\int_{t}^{u} r_{s} d s} d F_{u}=\int_{t}^{T} e^{-\int_{t}^{u} r_{s} d s} d F_{u}^{\tau_{n}}
$$

and it can be shown (see Karatzas and Shreve 1998) that the stopped process $F^{\tau_{n}}$ is a martingale. Thus, by Lemma 4.1 we get that the stochastic differential

$$
e^{-\int_{t}^{u} r_{s} d s} d F_{u}^{\tau_{n}}
$$


is a martingale differential, and we obtain

$$
\begin{aligned}
& E^{Q}\left[e^{-\int_{t}^{\tau_{n}} r_{s} d s}\left(F_{\tau_{n}}-X_{\tau_{n}}\right)-\int_{t}^{\tau_{n}} e^{-\int_{t}^{u} r_{s} d s} d F_{u} \mid \mathcal{F}_{t}\right] \\
& =E^{Q}\left[e^{-\int_{t}^{\tau_{n}} r_{s} d s}\left(F_{\tau_{n}}-X_{\tau_{n}}\right) \mid \mathcal{F}_{t}\right] .
\end{aligned}
$$

From the definition of $\tau_{n}$ we then have

$$
\begin{aligned}
& E^{Q}\left[e^{-\int_{t}^{\tau_{n}} r_{s} d s}\left(F_{\tau_{n}}-X_{\tau_{n}}\right) \mid \mathcal{F}_{t}\right] \geq E^{Q}\left[e^{-\int_{t}^{\tau_{n}} r_{s} d s}\left(\left[1-\frac{1}{n}\right] X_{\tau_{n}}-X_{\tau_{n}}\right) \mid \mathcal{F}_{t}\right] \\
& =-\frac{1}{n} E^{Q}\left[e^{-\int_{t}^{\tau_{n}} r_{s} d s} X_{\tau_{n}} \mid \mathcal{F}_{t}\right]
\end{aligned}
$$

Furthermore we have

$$
\begin{aligned}
& E^{Q}\left[e^{-\int_{t}^{\tau_{n}} r_{s} d s} X_{\tau_{n}} \mid \mathcal{F}_{t}\right] \leq e^{c(T-t)} E^{Q}\left[X_{\tau_{n}} \mid \mathcal{F}_{t}\right] \leq \frac{1}{1-1 / n} E^{Q}\left[F_{\tau_{n}} \mid \mathcal{F}_{t}\right] \\
& =e^{c(T-t)} \frac{F_{t}}{1-1 / n} .
\end{aligned}
$$

where we again have used the martingale property of the stopped process $F^{\tau_{n}}$. We thus have

$$
E^{Q}\left[e^{-\int_{t}^{\tau_{n}} r_{s} d s}\left(F_{\tau_{n}}-X_{\tau_{n}}\right)-\int_{t}^{\tau_{n}} e^{-\int_{t}^{u} r_{s} d s} d F_{u} \mid \mathcal{F}_{t}\right] \geq-e^{c(T-t)} \frac{F_{t}}{n(1-1 / n)}
$$

which tends to zero as $n \rightarrow \infty$.

We now turn to the uniquenesss proof and for this we consider again a fixed $t$ and define for each $n$ the stopping time $\tau_{n}$ by

$$
\tau_{n}=\inf \left\{s \geq t ; V_{s} \geq Z_{s}-\frac{1}{n}\right\} .
$$

Since $V^{\tau_{n}}$ is a martingale on $[t, T]$ and since $V$ is given by (52) it now follows that $F^{\tau_{n}}$ is a martingale on the same interval. By definition of $\tau_{n}$ it follows that

$$
e^{-\int_{t}^{\tau_{n}} r_{s} d s}\left(X_{\tau_{n}}-F_{\tau_{n}}\right) \leq 1 / n
$$

so we have

$$
X_{\tau_{n}} \leq F_{\tau_{n}}+\frac{1}{n} \cdot \frac{B_{\tau_{n}}}{B_{t}}
$$

Now assume that $G$ is a submartingale dominated by $X$. We then obtain

$$
\begin{aligned}
G_{t} & \leq E^{Q}\left[G_{\tau_{n}} \mid \mathcal{F}_{t}\right] \leq E^{Q}\left[X_{\tau_{n}} \mid \mathcal{F}_{t}\right] \\
& \leq E^{Q}\left[F_{\tau_{n}} \mid \mathcal{F}_{t}\right]+\frac{1}{n} \cdot E^{Q}\left[\frac{B_{\tau_{n}}}{B_{t}} \mid \mathcal{F}_{t}\right] \\
& =F_{t}+\frac{1}{n} \cdot E^{Q}\left[\frac{B_{\tau_{n}}}{B_{t}} \mid \mathcal{F}_{t}\right] \leq F_{t}+\frac{1}{n} \cdot \frac{C_{t}}{B_{t}}
\end{aligned}
$$

where $C$ is given by (11). Letting $n \rightarrow \infty$ gives us $G_{t} \leq F_{t}$ and we are done. 


\section{References}

[1] Boyle, P. (1989): The quality option and timing option in futures contracts. Journal of Finance 44, 101-113.

[2] Cohen, H. (1995): Isolating the wild card option. Mathematical Finance $5,155-165$.

[3] Gay, G., And Manaster, S. (1984): The quality option implicit in futures contracts. Journal of Financial Economics 13, 353-370.

[4] Gay, G., and Manaster, S. (1986): Implicit delivery options and optimal delivery strategies for financial futures contracts. Journal of Financial Economics 16, 41-72.

[5] Karatzas, I., And Shreve, S. (1998): Methods of Mathematical Finance. Springer.

[6] Protter, P.E. (2004): Stochastic Integration and Differential Equations. Springer, Second Edition.

[7] Revuz, D., ANd Yor, M. (1994): Continuous martingales and Brownian motion. Springer.

[8] Snell, J. (1952): Application of martingale system theorems. Trans. Am. Math. Soc. 73, 293-312. 